\title{
Laplace's demon tries on Aristotle's cloak: on two approaches to determinism
}

\author{
Tomasz Placek ${ }^{1}$
}

Received: 15 December 2015 / Accepted: 4 July 2016 / Published online: 16 July 2016

(C) The Author(s) 2016. This article is published with open access at Springerlink.com

\begin{abstract}
The paper describes two approaches to determinism: one focuses on the features of global objects, such as possible worlds or models of a theory, whereas the other's concern is the possible behaviour of individual objects. It then gives an outline of an individuals-based analysis of the determinism of theories. Finally, a general relativistic spacetime with non-isometric extensions is described and used to illustrate a conflict between the two approaches: this spacetime is indeterministic by the first approach but deterministic by the second approach.
\end{abstract}

Keywords Determinism and indeterminism - General relativity · Initial value problem

\section{Introduction: two traditions of thinking about determinism}

There are two traditions of thinking about determinism, one centred upon individual objects and the other centred upon an entire universe. The former tradition focuses on relatively small objects or processes (that is, small if compared with the universe) and asks if these objects or processes could evolve differently than they actually did. The cloak story that Aristotle tells in De Interpretatione clearly exemplifies this way of thinking: a given cloak might wear out, but it could be cut up first, that is, before wearing out. As we deliberate whether or not the example argues in favour of indeterminism, the data we look at are limited in space and time. It is of course the cloak that matters, but some of its surroundings are relevant as well. Just think of a particular person who

\footnotetext{
$\triangle$ Tomasz Placek

tomasz.placek@uj.edu.pl

http://www.iphils.uj.edu.pl/branching

1 Department of Philosophy, Jagiellonian University, 52 Grodzka room 17, 31-044 Kraków, Poland
} 
is a potential cutter, a knife, the particular material and weather conditions that make cloth wear out in time, but also make it capable of being cut up. However large these surroundings are, if compared to the cloak, we typically do not extend them to the entire universe. That is, typically we are happy to limit the data for the determinism question to a relatively small region of our spatiotemporal universe. This observation applies as well to another great picture we owe to Aristotle, that of tomorrow's sea battle. Although armadas of military vessels, together with sailors, their commanders, weather conditions, etc., occupy a relatively large area of the sea, this span is just a tiny spatiotemporal region of our entire world. In some examples used in this tradition, namely those involving human agents and their decisions, the data is even further restricted-to a particular person, or more precisely, to some particular period in that person's life. In this vein, to illustrate future contingency and motivate his three-valued logic, Łukasiewicz (1961) invokes a certain Piotr, who may come to the Castle Square in Warsaw tomorrow but as well may not come to the said location tomorrow. ${ }^{1}$ A clear statement of this individuals-based approach to determinism is in Prior (1962, p. 59): ${ }^{2}$

[...] [I]t seems perfectly possible to say that some things, but not all things, have alternative possibilities of reaction to one and the same stimulation. It is "open," we might say, to a disturbed electron to jump to orbit $A$, and equally open to it to jump to orbit $B$, but perhaps not open to it to jump to orbit $C$. In other words, its dispositions may be such that with certain provocations it will "jump to orbit $A$ or to orbit $B$ " without having any determinate disposition to jump to orbit $A$, or any to jump to orbit $B$. [...] Persons, say, have the power, without the necessity, of doing $X$ in certain circumstances; for oysters, on the other hand, doing $X$ may be necessary or impossible; and $Y$, say turning into a dragon, may be something which no existing object has the power to do [...].

Judging by examples used in the literature, this local approach to determinism and future contingents is a characteristic feature of theories of agencies and modal logic, in particular that branch of logic that investigates the combination of tense and modality. Philosophers' debates aside, it is this approach that is used in mundane and everyday's contexts, in science labs for instance. A chemistry student investigating a catalytic reaction may wonder, seeing different outputs of seemingly the same process in subsequent runs of her experiment: is the varying output due to the indeterministic nature of the process, or to some differences in the reaction's initial conditions in subsequent runs of the experiment? In an attempt to clarify the issue, she focuses on local matters of fact: is the catalyst, as well as other chemical substances used, sufficiently similar in subsequent runs of the reaction? Are the temperature, pressure, concentrations, and other relevant characteristics the same in all these runs? The universe and its possible evolutions are not part of the equation.

The second tradition, one that centres upon most global notions like that of the universe, world or history, is invariably linked to Laplace's vision. In Laplace's well known metaphor, a super intelligence is capable of "seeing" the entire past and future

\footnotetext{
1 That paper consists of a much earlier text that was read as Łukasiewicz's opening address as the Rector of the University of Warsaw in 1922.

2 I am indebted to Jacek Wawer for drawing my attention to this paper.
} 
of the universe, thanks to its grasp of an instantaneous state of the Universe and its knowledge of all of the forces acting in the Universe. After the removal of epistemic overtones, signalled by words like "knowledge", or "seeing", the vision forms the backbone of the current received analysis of the determinism of theories. The basic intuition of this approach is that of "once similar, always similar", that is, a theory is deterministic iff whenever two models of the theory agree on their initial segments, they agree as wholes. To make the idea precise, three notions are required, namely (isolated) systems of a theory, models of a theory, and isomorphism (similarity) between segments of a theory's models. As for the first notion, one begins with asking what the world would be like, if a theory considered were true. In addressing this question, one considers systems that fall in the scope of a given theory, and assumes that such systems are isolated. To achieve the isolation, a theory's system is viewed as completely separate, as a (toy) possible world (cf. Butterfield 2005). As for the notion of models, contrary to appearances, it should not be confused with models in the sense of logic. However, in spacetime theories models can be identified with appropriate differential manifolds with some objects defined on them (cf. Earman 2008). The notion of isomorphism is called for in order to clarify the basic intuition of the universe-based approach that determinism means that the similarity of the initial segments of models implies the similarity of whole models. Isomorphism needs to be a relation less strict than identity since models can differ by merely some mathematical surplus structure, while representing the same physical states. ${ }^{3}$ However, since theories are not understood in this approach as formal theories, and a theory's models are not simply models in the sense of logic, isomorphism cannot be a standard notion as defined in logic [i.e., as a relation between models of a formal language, cf. Hodges (1993)]. One could think perhaps of this notion as relative to the mathematical structure of a given theory: given a mathematical structure of a theory, an appropriate notion of isomorphism will be definable. An example is general relativity in which isomorphism is defined in terms of diffeomorphism between smooth manifolds. ${ }^{4}$

Having introduced the required notions, we quote one formulation of the received analysis of determinism:

Determinism is [...] a matter of isomorphic instantaneous slices implying that the corresponding final segments are isomorphic (where 'corresponding' means 'starting at the time of the instantaneous slice'). That is: we say that a theory is deterministic if, and only if: for any two of its models, if they have instantaneous slices that are isomorphic, then the corresponding final segments are also isomorphic. (Butterfield 2005)

Drawing a distinction between the two notions of determinism (and indeterminism) would be merely an exercise in pedantry if they agreed in their verdicts. But, emphatically, they do not agree. In the next Sect. 2 we will discuss two cases, known from the literature, in which the two approaches disagree. We will then sketch an analy-

\footnotetext{
3 This worry is not merely theoretical, since theories with gauge freedom (quite typical in physics) exhibit exactly this feature.

${ }^{4}$ For technical details of the received analysis of determinism as well as a survey of variants of this analysis, see Müller and Placek (2016).
} 
sis of individuals-based determinism in Sect. 3. Next we will proceed in Sect. 4 to a case little discussed in philosophical literature, that is, non-isometric extensions of spacetimes of general relativity (GR). The case is classified as indeterministic by the universe-based approach whereas the individuals-based approach yields the verdict of determinism. The paper ends with Sect. 5 .

\section{On space-invaders and buckling columns}

Although analyses of determinism that appeal to possible worlds or models of a theory are dominant in the literature, there have been a few attempts to define determinism in terms of the behaviour of individual objects. The motivation for these attempts was provided by systems for which the received analysis of determinism deliver counterintuitive verdicts. We discuss below two systems of this sort, a system with a space invader and a buckling column.

\subsection{Space-invaders}

What is referred to as a space-invader is a system of Newtonian gravitation whose essence is quite simple: a particle does not exist before time $t_{0}$ and at this time it emerges from spatial infinity, and continues to exist afterwards. A system of this sort is exemplified by Xia's (1992) system of five masses interacting by the force of Newtonian gravitation. Given specific initial conditions, one of these masses escapes to spatial infinity at some (finite) time $t_{0}$. Since the theory of Newtonian gravitation respects the time-reversal symmetry, a system symmetrical to the above, that is, one with the fifth mass emerging from infinity at time $-t_{0}$, counts for a system of Newtonian gravitation.

Intuitively speaking, since before $-t_{0}$ there is absolutely no factor indicating that the particle will come to being, and if so, when this will happen, the system seems to be indeterministic. Let us call the intuition underlying this sentiment an "indeterminacy intuition". In contrast to the indeterminacy intuition, if we focus on each individual mass, we see that as soon as it exists, it does not exhibit any trace of having alternative possible evolutions. Building on the indeterminacy intuition, one envisages many alternative evolutions of the spatiotemporal universe. These evolutions share some initial segment (one with four existent masses), but differ with respect to when the fifth mass comes to being. Among these evolutions, there is also one with no fifth mass ever. In contrast, the individuals-based approach invites one to posit just one possible evolution, one in which the fifth mass comes to being at the specified time $-t_{0}$ (remember that $t_{0}$ is determined by the initial conditions of the system we started with). The proponent of individuals-based approach thus refuses to read the indeterminacy intuition as hinting at indeterminism, since no individual object of the system has alternative possible evolutions.

It is controversial which of the two diagnoses is more adequate, and this controversy reflects, I believe, the fact that there are two notions of determinism at hand, and the context of the debate does not indicate which one is to be to used. 


\subsection{A buckling column}

A buckling column is a system that has some symmetry at an initial phase, and because of that symmetry tends to be classified as deterministic by the received analysis of determinism, whereas it seems to be indeterministic (intuitively speaking). This particular system is just an example of an initially symmetric system that can be used to impugn Lewis's (1983) analysis of determinism. A buckling column is a rotationally symmetric system consisting of a slim column in a uniform gravitational field and under a weight placed symmetrically on the column's top. To emphasise the system's crucial features, its rotational symmetry is perfect and the weight is critically heavy, i.e., the column cannot retain its shape, it must fall. Systems of this sort are studied in the elasticity theory, see e.g., Al-taee (2010).

Objections to the received analysis of determinism are based on a perception that (1) the column will buckle, and, because of the symmetry of its initial stage, i.e., before the buckling, (2) the buckling can be in any direction. For a record, it should be mentioned, however, that the first part of this diagnosis is not universally accepted. Given the perfect symmetry of the system before the collapse, it might be argued that the symmetry should persist after the collapse, which means that the column will not buckle but become symmetrically flattened by the weight on its top. One might argue against this view by appealing to the elasticity theory. Without actually solving the dynamical equation (i.e., by providing exact initial conditions) for a given system, the elasticity theory investigates the form of the state function of the column, asking how this function behaves, i.e., where its minima, maxima, and saddle points are. The analysis of a buckling column in two dimensions (such a column can buckle in one of only two directions) says that there are two stable solutions for the column, identified with buckling in the two directions, and one unstable solution, identified with the symmetrical flattening of the column..$^{5}$ This result, however, allows for different interpretations. One interpretation claims that there are only two possible directions of buckling, classifying the unstable solution as impossible. On an alternative interpretation, the stable solutions are identified with solutions resulting from perturbed (i.e., slightly non-symmetrical) initial states, an unstable solution-with the evolution developing from a perfectly symmetrical initial state. This latter interpretation thus says that given the ideal symmetry of the initial state, only one evolution, i.e., one without buckling, is possible.

It thus seems that to use the buckling column in an argument for inadequacy of Lewisian analysis of determinism, we need to consider a world with physics slightly different from Newtonian gravitation, just different enough so that perfectly symmetric columns buckle there. ${ }^{6}$ Then, the argument goes, by the symmetry of the column's pre-fall stage, there are plenty of such possible worlds, each containing a column buckling in a (seemingly) different direction.

\footnotetext{
5 We mention the analysis in 2 dimensions, as it is mathematically tractable —cf. Rosales (2012).

6 This move is taken by Belot (1995).
} 
Now, Lewis's definition as well as the received view, opt for a relation less stringent than identity to match segments of possible worlds (or segment of a theory's models). ${ }^{7}$ For the received view, the relation is that of isomorphism, and what this exactly means depends on the mathematical structure of a theory at hand. Lewis prefers the relation of "duplication" that is to be defined in terms of qualitative and "perfectly natural" properties and relations. Given that he takes the latter to be discoverable by science, there is little harm, I believe, in identifying his "duplication" with "isomorphism" of philosophers of science.

Turning finally to the argument, consider two models (of a slightly changed Newtonian gravitation), in which the column buckles in different directions. For the two models to be a witness for indeterminism, they should be non-isomorphic but contain isomorphic initial segments. Clearly, the initial, that is, pre-buckling, segments of these models are isomorphic; are then the whole models non-isomorphic? Well, one model is the transform of the other by a rotation over some angle. Naturally, the two models must then be isomorphic. After all, if rotational symmetry did not count as isomorphism, what then would? Consequently, the column is classified as a deterministic system.

A natural idea of how to improve on the received analysis of determinism, pursued by Belot (1995), Melia (1999), and Sattig (2015), is to take into account possible evolutions of individual objects comprising the system. The column may fall in different directions, because in one possible scenario a particular individual object lands on the top surface of a fallen column, and in another possible scenario-on the bottom surface of the fallen column. A pair of models in which not one particle, but two particles, occupy the above "alternative" positions (one on the top, the other on the bottom, surfaces of the fallen column) seems to be a wrong representation of the modal situation at hand. The feeling is that in such a case a global isomorphism relating whole models betrays local isomorphism relating the initial segments, as the latter pairs the particles that are not paired (identified) by the former. These ideas are translated into this definition, due to Belot (1995): ${ }^{8}$

$W$ is deterministic if, whenever $W^{\prime}$ is physically possible with respect to $W$, and $t, t^{\prime}$, and $f: W_{t} \rightarrow W_{t^{\prime}}^{\prime}$ are such that $f$ is an [isomorphism] [...], there is some [isomorphism] [...] $g: W \rightarrow W^{\prime}$ whose restriction to $W_{t}$ is $f$.

Thus, to prove that $W$ is indeterministic, we need to find $W^{\prime}$ physically possible with respect to $W$, times $t, t^{\prime}$, initial segments $W_{t}, W_{t^{\prime}}^{\prime}$ of $W, W_{t^{\prime}}^{\prime}$, resp., and a local isomorphism $f: W_{t} \rightarrow W_{t^{\prime}}^{\prime}$ such that for every global isomorphism $g: W \rightarrow$ $W^{\prime}, f \neq g_{\mid W_{t}}$. Let us thus see how this definition works in the case of a buckling column. We would like to prove that any global isomorphism pairs constituents of the two columns badly, i.e., identifies those constituents that a local isomorphism does not

\footnotetext{
7 Lewis's (1983) reasons for rebuking the use of identity in this context are different from those of philosophers of physics, though, and stem from his rejection of overlapping possible world in favour of the duplication of possible worlds.

8 In the quote "duplication" is replaced by "isomorphism", which may misrepresent Lewis's idea, as duplication might be seen as pairing both individuals and individuals' states, whereas isomorphism is concerned with individuals' states only—see more about it later.
} 
identify. But we shall see that the argument does not go through—unless isomorphism relates bare particulars.

Let symbols with a prime sign refer to objects in $W^{\prime}$, and symbols without a prime sign-to objects in $W$. Let $\alpha(x)$ stand for a complete state, defined in terms of perfectly natural properties and relations, of object $x$ in the pre-fall stage $W_{t}$ of $W$. Similarly, let $\beta(y)$ stand for a complete state of object $y$ in the post-fall segment $W \backslash W_{t}$ of $W$. To draw attention to a state at a moment $m$ of time, we will use subscript, like in $\alpha_{m}(x)$. And $t$ and $t^{\prime}$ refer to moments of buckling in the respective models.

One might naturally want to take for $f$ the identity, but this is excluded as the worlds are not allowed to overlap. Since the pre-fall segments are rotationally symmetric, there is freedom which object $x$ should be paired with which object $x^{\prime}$ by the relation on states, i.e., $f(\alpha(x))=\alpha^{\prime}\left(x^{\prime}\right)$. After all, for every $x_{1}$ that is the transform of $x$ by some rotation $O_{\Theta}, \alpha(x)=\alpha\left(x_{1}\right)$. (An analogous condition holds for states of constituents of $W^{\prime}$.) It follows that $f$ must preserve the rotational symmetry, in the following sense:

If $f(\alpha(x))=\alpha^{\prime}\left(x^{\prime}\right)$, then for every $x_{1}$ such that $x_{1}=O_{\Theta}(x)$ for some angle

$\Theta, f\left(\alpha\left(x_{1}\right)\right)=\alpha^{\prime}\left(x^{\prime}\right){ }^{9}$

We thus pick an isomorphism $f$ that preserves rotational symmetry; clearly, it pairs point particles $x$ and $x^{\prime}$ that lie on the side surfaces of the respective columns. Suppose that in post-fall segments, $x$ and $x^{\prime}$ land in different states in corresponding moments $m, m^{\prime}$, say, one is on the top surface, while the other-on the bottom surface, of the fallen columns. ${ }^{10}$ Then for every global isomorphism $g, g\left(\beta_{m}(x)\right) \neq \beta_{m^{\prime}}^{\prime}\left(x^{\prime}\right)$. So, whatever the global isomorphism $g$ is, it must pair state $\beta_{m}(x)$ of $x$ with a state of some $y^{\prime}$ that is not the same as $x^{\prime}$, i.e., ${ }^{11}$

$$
g\left(\beta_{m}(x)\right)=\beta_{m^{\prime}}^{\prime}\left(y^{\prime}\right) \text { where } y^{\prime} \neq x^{\prime} .
$$

We would like to argue that not only for post-fall states, $x$ and $y^{\prime}$ are related as above, but they are so related in the pre-fall segments. That is, to argue that $g$ restricted to $W_{t}$ is not identical to $f$, i.e., $g\left(\alpha_{z}(x)\right) \neq f\left(\alpha_{z}(x)\right)$ for some moment $z<t$, we need:

$$
g\left(\alpha_{z}(x)\right)=\alpha_{z^{\prime}}^{\prime}\left(y^{\prime}\right) \text { for some corresponding moments } z<t \text { and } z^{\prime}<t^{\prime} .
$$

Note, however, that both $x^{\prime}$ and $y^{\prime}$ lie on the side surface of the column in $W^{\prime}$, and although in the post-fall segment they occupy alternative positions (top vs. bottom), in the pre-fall segment they are related by some rotational symmetry, $x^{\prime}=O_{\Theta^{\prime}}^{\prime}\left(y^{\prime}\right)$, so at any moment $z^{\prime}<t^{\prime}$, their states must be the same, $\alpha_{z^{\prime}}^{\prime}\left(x^{\prime}\right)=\alpha_{z^{\prime}}^{\prime}\left(y^{\prime}\right)$. Accordingly, we get:

\footnotetext{
9 One consequence of this condition is that point particles equidistant from the axis of one column will be paired to point particles equidistant from the axis of the second column.

10 As the two columns fall in different directions, there must be some difference between $x$ and $x^{\prime}$ in the post-fall segments; for brevity we use the mentioned difference in location.

11 If $x$ landed on the top surface, an isomorphism should relate it to some particle on the top surface as well, so it cannot be $x^{\prime}$, as it landed on the bottom surface.
} 


$$
g\left(\alpha_{z}(x)\right)=\alpha_{z^{\prime}}^{\prime}\left(y^{\prime}\right)=\alpha_{z^{\prime}}^{\prime}\left(x^{\prime}\right)=f\left(\alpha_{z}(x)\right) .
$$

Thus, in contrast to the motivation underlying Belot's definition, we obtained that the restriction to $W_{t}$ of any global isomorphism operative in the buckling column example is identical to $f$, so the buckling column is, after all, deterministic. Has anything gone wrong in the reasoning above? One construal of the duplication relation (recall that Belot uses duplication rather than isomorphism) is that it affords the pairing of objects in possible worlds that goes over and above quantitative descriptions of these objects. Technically, the domain as well as the range of a duplication are, on this construal, sets of constituents of possible worlds. Then it is immediate to see that in the buckling column example, for some local duplication $f$, there is no global duplication $g$ that extends $f$. Just take $f$ that pairs, by the formula $f(x)=x^{\prime}$, objects $x$ and $x^{\prime}$ that in the post-fall segments land on the opposite sides of the fallen columns (top vs. bottom). Any global duplication must thus relate $x$ (on the top of the first column) with some $y^{\prime} \neq x^{\prime}$ (on the top of the second column), i.e., $g(x)=y^{\prime}$. Since $y^{\prime} \neq x^{\prime}$, it is not the case that $g_{\mid W_{t}}=f$.

In order for the relations of duplication or isomorphism to do the required job in the discussed definition of determinism, they need to relate bare particulars. I take this as an argument against the definition. Now, in contrast to the motivation underlying this definition, individual objects are little present in it, as the job is done by local and global isomorphisms (duplications). Shouldn't thus individual objects themselves play a more distinctive role in an analysis of determinism? A firm step in this direction that avoids reference to duplications or isomorphisms, is Sattig's (2015, p. 5) notion of "Strong Qualitative Determinism":

A possible world $w$ is deterministic iff for all times $t$, and for all objects $x$ in $w$, there is no object in any possible world with the same laws of nature as $w$, which matches $x$ in its qualitative description up to $t$, but which does not match $x$ in its total qualitative description.

Applying this definition to the buckling column, possible worlds with buckling columns that we discussed have the same laws of nature. Let us focus on one such a world, and pick there a particle $x$ on a column's surface that ends up on the bottom surface after the fall. It is easy to think of another world and a particle in it whose qualitative description before the fall matches the qualitative description of $x$ before the fall, but which does not land on the bottom surface after the fall. The definition thus yields a verdict of indeterminism for the buckling column.

Sattig's analysis still appeals to a Lewisian picture of non-overlapping worlds, so to decide on determinism two objects are needed, each inhabiting a different possible world, rather than just one object. This appeal to two objects is not natural-typically, one relates indeterminism to one object facing alternative possible future continuations. Moreover, this intuition finds support in the treatment of the initial value problem, which is the main source of information on the determinism and indeterminism of theories of physics. One reason that the initial value problem is not well-posed is that for given initial conditions there is more than one solution to a theory's basic equations. These are solutions for one system and they diverge despite being identical for some arguments, which invokes an image of branching. In what follows we will thus opt for 
a branching-style version of Sattig's analysis, in which one and the same individual object faces alternative evolutions, and accordingly, possible worlds overlap. But it is worth noting that Sattig's analysis as well as our approach give similar diagnoses of our main example, the non-isometric extensions of a GR spacetime.

\section{An outline of individuals-based determinism}

To sketch our individuals-based approach to the determinism of scientific theories, we begin with an observation that a theory typically comes with a verdict regarding what its individual objects are (individual objects are somewhat localised in space and time, are bearers of states, and stand in relations to themselves and other entities posited by the theory). Since some theory may admit no individual objects, a verdict might be purely negative. In some cases, the question of what are individual objects is not resolved by a theory itself, but left for the theory's interpretations.

Having the notion "individual objects admitted by a theory, under a given interpretation" we ask next how a given theory describes the evolution of the individual objects it admits. Is an object's evolution unique in the whole time considered? Or perhaps, it is unique but only locally, that is, in an arbitrary small neighbourhood around each set of allowable initial conditions, but globally non-unique? Or does the uniqueness of evolutions fail already at the local level? These questions might turn out to be dauntingly hard, but one does not need anything over and above the mathematical apparatus of a theory to address them. In the spirit of the individuals-based approach, as the first approximation, a theory is judged deterministic if each individual object it admits is described by this theory as having a globally unique evolution for each allowable set of initial conditions. Otherwise, the theory is judged to be indeterministic. Note, however, that a final analysis needs to take into account the fact that multiple evolutions of states admitted by a theory does not necessarily mean multiple evolutions of "real" states, as the relation between states posited by a theory and "real" states might be many-to-one. Thus, only multiple and dissimilar evolutions count as evidence for indeterminism. The notion of dissimilarity is to be explained in terms of a theory's gauge freedom, which is an issue decided by a theory (or a theory's interpretation).

The determinism question, as described above, looks very much as one for practitioners to decide, since they know the mathematical details and interpretational subtleties of a theory best to address the question of the uniqueness and the existence of solutions to the theory's basic equations. From the perspective of the philosophy of science, however, this description of determinism is still off the mark: we have not provided a semi-formal analysis of the determinism of theories that could be applicable to all, or most, scientific theories. It is this task that philosophers of science strive to achieve, and to which we now turn.

The definition of the determinism of scientific theories that is tailored to accommodate the possible behaviour of individual objects is that of Müller and Placek's (2016); here we only describe its essential ingredients, referring the reader interested in the technical details to the aforementioned paper. Recall that in the received analysis of determinism, the crucial notion is that of the model of a theory. What is needed in the present approach is its analogue, the modal representation of a system of a theory. 
A distinctive feature of the present approach is that it requires a means to represent alternative possible evolutions of an individual object (as well as of the entire world), as conceived by a theory at hand. Such a means - a modal representation of a theory's system-is thus modally thick, if the theory considered allows for alternative possible evolutions, i.e., by having multiple dissimilar solutions to its basic equations (for the same initial conditions). Consequently, a theory's indeterminism is an internal feature of its modal representation. This is in stark contrast to the received view on determinism, as the notion of models it uses is modally thin: a model is not capable, on its own, of representing alternative possibilities; determinism (and its negation) are features of a class of models rather than of a single model.

A choice of a modally thin concept of model over a modally thick concept of modal representation is driven by metaphysical preferences. The choice reflects the old debate between Humeans and anti-Humeans: does the world, or a model of the world, comprise only those things that actually happen, or does it accommodate things as well that might have happened, or may happen in the future? I surmise that for a full-fledged notion of indeterminism, one needs real possibilities; hence modal representations offer a more adequate format than modally thin models. ${ }^{12}$

What is then a modal representation of a system of a given theory? In essence, it is the set of (overlapping) dissimilar solutions to the theory's basic equations, for a system of that theory. Accordingly, if a theory permits just one solution for a system considered, its modal representation contains only one solution. But a modal representation can contain many overlapping solutions. For an example, consider the system of Norton's (2008) dome, which is a point mass located on the top of a particularly shaped dome, in a uniform gravitational field. The system's modal representation is constructed from the set of functions $r_{b}: \Re \rightarrow \Re, b \in \Re^{+} \cup\{\infty\}$, each representing the point mass's position in time, as being on the top of the dome till time $b$, and sliding down its slope after $b .{ }^{13}$ In the the construction, the set of solutions for the system is redescribed, with the aim of disclosing two features: (1) the ordering inherent to the spatio-temporal (temporal) and modal structure of the solutions, and (2) the assignment of states. In the example of Norton's dome, the resulting ordering is a tree-like partial ordering of possible moments of the system (aka branching-time, BT), ${ }^{14}$ and the assignment relates possible moments to positions of the point mass [for details, see Müller and Placek (2016)].

The ordering part of a modal representation depends on the kind of theory considered. For all non-relativistic theories, it is the BT ordering. Special relativity and some GR spacetimes require the ordering of branching spacetimes, [BST, see Belnap (1992)]. In general, GR spacetimes (including those with closed time-like curves) seem to call for a "patch-wise" BST ordering that I investigated in Placek (2014).

\footnotetext{
12 For a discussion of modal notions, and Humean notions, of determinism, see Placek et al. (2014).

13 Observe that a system's description which require the point mass to be on the dome's top since $-\infty$ in time makes all the solutions with $b \neq \infty$ similar, as they all are related one to the other by the timetranslation symmetry. A different description of the system does not imply the time-translation symmetry, however.

14 "Tree-like" means that the ordering has a single trunk and there is no backward branching, i.e., $(x \leqslant$ $z \wedge y \leqslant z) \rightarrow(x \leqslant y \vee y \leqslant x)$.
} 
The definition of determinism is particularly simple if the ordering part of modal representations is BT [this option is explored in detail in Müller and Placek (2016)]. Maximal chains of moments (i.e., of simultaneity slices) are taken for maximal modally consistent subsets of the ordering, and interpreted (after an assignment of states) as possible histories. A modal representation is said to be indeterministic iff it contains more than one possible history. A theory is indeterministic iff some system of that theory has an indeterministic modal representation. Once a concept of modal consistency for other relevant orderings (i.e., other than BT) is agreed upon, ${ }^{15}$ the above definition carries over to theories with modal representations that are not based on BT.

Reflecting on the above analysis, to claim the indeterminism of a modal representation, in its ordering part one needs to find what Placek et al. (2014) called a "modal fork", i.e., a fork whose arms are contained in alternative histories. A modal fork thus signals alternative possible evolutions. In a search for cases of individual-based indeterminism, one needs thus to attend to modal forks resulting from the behaviour of individual objects. The recipe will not work for theories, the modal representation of which involves the BT ordering, since BT is too frugal to capture the world's spatial aspect, and hence cannot represent individual objects.

\section{A GR system: indeterministic universe-wise but deterministic individuals-wise}

We are now about to show that the distinction between universe-based determinism and individuals-based determinism matters: we will exhibit a GR system that is indeterministic universe-wise by the received analysis, but is deterministic individuals-wise. The problem is known as non-isometric extensions of GR spacetimes. In philosophical literature non-isometric extensions of some GR spacetimes were described in Earman (1995) and Belot (2011); the latter takes them as evidence for indeterminism of GR.

\subsection{Technical prerequisites}

Let us review the required terminology. ${ }^{16}$ An $n$-dimensional GR spacetime is a pair $\left\langle M, g_{a b}\right\rangle$, where $M$ is a connected $n$-dimensional Hausdorff manifold (without boundary) that is infinitely differentiable (smooth) and $g_{a b}$ is a smooth, non-degenerate, pseudo-Riemannian metric of Lorentz signature $(+,-, \ldots,-)$ defined on $M$. Manifold $M$ is Hausdorff iff it satisfies the Hausdorff property, which says that for every pair $\left\langle x_{1}, x_{2}\right\rangle$ of distinct points in $M$ there are non-overlapping open subsets $O_{1}$ and $O_{2}$ of $M$ such that $x_{i} \in O_{i}(i=1,2)$. It is said that two spacetimes $\left\langle M, g_{a b}\right\rangle$ and $\left\langle M^{\prime}, g_{a b}^{\prime}\right\rangle$ are isometric if there is a diffeomorphism (smooth bijection) $\varphi: M \rightarrow M^{\prime}$ such that the induced pull-back function $\varphi^{*}$ satisfies $\varphi^{*}\left(g_{a b}^{\prime}\right)=g_{a b}$. A spacetime $\left\langle M^{\prime}, g_{a b}^{\prime}\right\rangle$ is an extension of $\left\langle M, g_{a b}\right\rangle$ if there exists an embedding $\Lambda: M \mapsto M^{\prime}$ (i.e.,

15 For a discussion of various relevant concepts of modal consistency, see Müller (2014).

16 Explanations of the mathematics of GR can be found in mathematically-oriented books on GR, like e.g., Malament (2012). 
$\Lambda$ is a diffeomorphism onto its image $)$ and $\Lambda^{*}\left(\left.g_{a b}^{\prime}\right|_{\Lambda(M)}\right)=g_{a b}$ and $\Lambda(M) \neq M^{\prime}$. A spacetime is maximal iff it has no extension.

With each point $p \in M$ there is an associated vector space, aka tangent space, $M_{p}$, on which $g_{a b}$ induces a cone structure, so that each vector $\xi^{a} \in M_{p}$ is either timelike, or null, or spacelike. Time-orientable spacetimes permit a distinction between future and past lobes of light-cones; technically, a time-orientable spacetime has a continuous timelike vector field on "its" manifold.

A smooth curve $\gamma: I \rightarrow M$ (where $I$ is an open interval of $\Re$ ) is timelike (resp., spacelike, or null) iff its tangent vector $\xi^{a}$ at each point in $\gamma[I]$ is timelike (resp., spacelike, or null). A curve is causal if its tangent vector at each point is either null or timelike. A curve is inextendible iff it has no endpoints. A geodesic in a spacetime $\left\langle M, g_{a b}\right\rangle$ is a curve $\gamma: I \rightarrow M$ that satisfies, for every vector $\xi^{a} \in M_{p}(p \in \gamma[I])$ tangent to the curve the condition $\xi^{a} \nabla_{a} \xi^{b}=0$, where $\nabla_{a}$ is the (unique) derivative operator compatible with $g_{a b}$. For any set $S \subseteq M$, the domain of dependence of $S$, written $D(S)$, is the set of points $p \in M$ such that every inextendible causal curve through $p$ intersects $S$. $S$ is an achronal subset of $M$ iff no two points in $S$ can be joined by a timelike curve.

\subsection{Determinism and the initial value problem in GR}

We need now to explain how non-isometric extensions of a GR spacetime are related to the determinism question. The determinism question presupposes a notion of a system evolving in time. This latter notion is typically not well-defined in general relativity, as usually a GR spacetime does not come with a distinguished time. Yet, a somewhat similar issue can be considered in GR: suppose we are given a 3-dimensional space with possibly some data on it. (Technically it is a manifold with a metric " "appropriate for space", i.e., a Riemannian metric). The question now is: can this space be uniquely extended to a 4-dimensional spacetime, i.e., to a manifold with the Lorentzian metric that satisfies the properties required from a manifold representing a GR spacetime (listed above), and in which Einstein field equations (EFE) hold? The answer crucially depends on the kind of data assumed on the space and on the properties the sought-for spacetime is supposed to have. As for the latter, the relevant factors is the existence of a matter field, and (if it is assumed to exists) the kind of model for the matter field; another issue is the occurrence of the cosmological constant in EFE. However, given the extension problem we will consider, a simple case is enough for our purpose. We focus upon spacetimes with a vanishing Ricci tensor, the so-called vacuum spacetimes and consider EFE without the cosmological constant. A satisfactory data for this case consists of a Riemannian metric $\tilde{g}$ and symmetric covariant 2-tensor $\tilde{k}$ that represents incremental changes of the metric in the direction normal to $\Sigma$. In this case the initial value problem amounts to constructing a 4-dimensional manifold $M$ with a Lorentz metric $g$ and an embedding $i: \Sigma \rightarrow M$ such that if $k$ is the second fundamental form on $i(\Sigma) \subset M$, then $i^{*}(g)=\tilde{g}$ and $i^{*}(k)=\tilde{k}$, where $i^{*}$ is the pull-back function induced by embedding $i$. Further, there is a set of equations relating $\tilde{g}$ and $\tilde{k}$, known as (vacuum) constraint equations, which guarantee the satisfaction of EFE in a sought- 
for spacetime. Space $\Sigma$ with the tensors $\tilde{g}$ and $\tilde{k}$ that satisfy the (vacuum) constraint equations are said to form a (vacuum) initial data set $\langle\Sigma, \tilde{g}, \tilde{k}\rangle$.

A result that is highly relevant to the initial value problem in the vacuum case has been obtained by Choquet-Bruhat and Geroch (1969) in the context of globally hyperbolic spacetimes. A hyperbolic spacetime has particularly nice causal properties. To recall, $\left\langle M, g_{a b}\right\rangle$ is said to be globally hyperbolic iff there is an achronal subset $S \subseteq M$, whose domain of dependence is the whole spacetime. One consequence of this definition is that a globally hyperbolic spacetime can be foliated by Cauchy surfaces (though the foliation is non-unique). Choquet-Bruhat and Geroch restrict their attention to globally hyperbolic spacetimes that could be developed from a given vacuum initial data set which (1) are vacuum solutions to EFE and (2) such that the image of space $\Sigma$ by the embedding is a Cauchy surface in $\langle M, g, k\rangle$. A spacetime satisfying these conditions is called a "vacuum Cauchy development" (VCD) of the initial data set. Note that condition (2) above implies that VCD is a globally hyperbolic spacetime. The theorem says:

Theorem 1 Let $\langle\Sigma, \tilde{g}, \tilde{k}\rangle$ be an initial vacuum data set. Then there is a unique, up to isometry, maximal VCD $\langle M, g, k\rangle$ of $\langle\Sigma, \tilde{g}, \tilde{k}\rangle$.

The phrase "unique up to isometry maximal VCD" means that if there is another maximal $\operatorname{VCD}\left\langle M^{\prime}, g^{\prime}, k^{\prime}\right\rangle$ of the same initial data set, then there is a time-orientation preserving isometry $\varphi: M \rightarrow M^{\prime}$. Thus, taking isometry for an identifier of vacuum spacetimes of GR (which is a typical move), the result ensures the uniqueness of maximal globally hyperbolic spacetimes compatible with vacuum initial data sets.

There has been a considerable controversy of how to interpret this theorem, which is directly related to the so-called Strong Cosmic Censorship Conjecture (SCCC). After all, the theorem concerns globally hyperbolic developments only: it puts no restrictions on other developments of an initial vacuum data set. This raises the question whether a maximal globally hyperbolic development of an initial data set can be further extended (of course, the resulting extension cannot be globally hyperbolic). SCCC addresses this question, claiming that "for generic initial data to Einstein's equations, the maximal globally hyperbolic development has no extension”. (Ringström 2009, p. 188). ${ }^{17}$

Non-globally hyperbolic spacetimes of GR typically involve causal anomalies, like closed timelike curves (CTC's); one might thus think, and this thought is the content of one interpretation of SCCC, that non-globally hyperbolic developments of initial data sets are not physical. An alternative interpretation of SCCC, and one used in recent research of the initial value problem in GR (cf. Ringström 2009) is that the non-globally hyperbolic developments of initial data sets are rare, in some measure-theoretical sense, with a measure defined on the space of relevant solutions to EFE. Without entering here a voluminous debate on SCCC, we opt for the latter interpretation, as there is arguably no data supporting non-physicality of the discussed solutions. In a slogan, "rare" does not mean "unphysical".

The Choquet-Bruhat and Geroch theorem has a consequence that evidence for indeterminism of GR (if there is one) in the vacuum case must consist of multiple non-isometric extensions of a maximal globally hyperbolic vacuum spacetime. By

\footnotetext{
17 The idea of SCCC is due to Penrose (1969); since then it has received many formulations.
} 
this theorem, an initial vacuum data set has a unique (up to isometry) maximal VCD, i.e., a maximal globally hyperbolic spacetime satisfying EFE. Thus, if this initial data set has multiple non-isometric developments satisfying EFE, these development must be non-isometric extensions of the maximal VCD determined by the data set. The problem of the determinism of GR (at least one related to the initial value problem) boils thus down to whether some maximal globally hyperbolic spacetime (satisfying EFE) has multiple non-isometric extensions (satisfying EFE).

\subsection{Non-isometric extensions of Taub spacetime}

We will describe below the construction of multiple non-isometric extensions of Taub spacetime. Our discussion is based on the paper of Chruściel and Isenberg (1993), which investigates as well a more realistic class of spacetimes, polarised Gowdy spacetimes, that have multiple non-isometric extensions. But since they are mathematically more demanding, we do here a simpler case of extensions of Taub spacetime.

Taub spacetime is a vacuum solution to EFE; the manifold is $M=\left(t_{-}, t_{+}\right) \times S^{3}$ and metric $g$ is given by

$$
d s^{2}=-U^{-1} d t^{2}+(2 l)^{2} U(d \psi+\cos \Theta d \varphi)^{2}+\left(t^{2}+l^{2}\right)\left(d \Theta^{2}+\sin ^{2} \Theta d \varphi^{2}\right),
$$

where and $m$ and $l$ are real positive constants, $\Theta, \varphi$ and $\psi$ are Euler coordinates on the 3-sphere $S^{3}, t_{ \pm}=m \pm\left(m^{2}+l^{2}\right)^{1 / 2}$, and

$$
U(t)=\frac{\left(t_{-}-t\right)\left(t-t_{+}\right)}{l^{2}+t^{2}} .
$$

Note that $U\left(t_{ \pm}\right)=0$, and hence the metric is not defined at $t_{ \pm}$. Taub spacetime is globally hyperbolic and maximally so, the Cauchy surfaces being identified by the condition $t=$ const for $t \in\left(t_{-}, t_{+}\right)$. As Newman, Tamburino and Unti (1963) showed, by using appropriate coordinate transformations $\langle M, g\rangle$ can be extended above $t_{+}$, the result being two non-hyperbolic spacetimes $\left\langle M^{\uparrow+}, g^{\uparrow+}\right\rangle$ and $\left\langle M^{\uparrow-}, g^{\uparrow-}\right\rangle$, known as Taub-NUT spacetimes. In a similar vein, Taub spacetime can be extended below $t_{-}$ into two non-hyperbolic spacetimes $\left\langle M^{\downarrow+}, g^{\downarrow+}\right\rangle$ and $\left\langle M^{\downarrow-}, g^{\downarrow-}\right\rangle$. Each $\left\langle M^{\uparrow+}, g^{\uparrow+}\right\rangle$, $\left\langle M^{\uparrow-}, g^{\uparrow-}\right\rangle,\left\langle M^{\downarrow+}, g^{\downarrow+}\right\rangle$, and $\left\langle M^{\downarrow-}, g^{\downarrow-}\right\rangle$ satisfies EFE and contains closed timelike curves in the region new with respect to $M$. As showed by Chruściel and Isenberg (1993), the pair $M^{\uparrow+}, M^{\uparrow-}$ and the pair $M^{\downarrow+}, M^{\downarrow-}$ are isometric.

To produce non-isometric extensions of Taub spacetime we need to glue together an upward extension together with a downward extension of Taub spacetime. "Gluing" means, in mathematical parlance, finding an equivalence relation $\equiv$ on the union of two manifolds, say $M^{\downarrow+} \cup M^{\uparrow-}$, and then taking the set of equivalence classes with respect to this equivalence relation. The result is the quotient structure $\left(M^{\downarrow+} \cup M^{\uparrow-}\right) / \equiv$.

Consider now four results of the gluing [for the equivalence relation used, consult Chruściel and Isenberg (1993, p. 1619)]:

$$
M^{a b}=\left(M^{\downarrow a} \cup M^{\uparrow b}\right) / \equiv \text {, where } a, b \in\{-,+\},
$$


each result being associated to metric $g^{a b}$, defined in terms of $g^{\uparrow a}$ and $g^{\downarrow b}$. Each $\left\langle M^{a b}, g^{a b}\right\rangle$ is a non-hyperbolic extension of Taub spacetime $\langle M, g\rangle$ and it satisfies EFE. As for isometries, there are the following results (1993, Theorem 3.1):

1. $\left\langle M^{+-}, g^{+-}\right\rangle$is isometric to $\left\langle M^{-+}, g^{-+}\right\rangle$.

2. $\left\langle M^{++}, g^{++}\right\rangle$is isometric to $\left\langle M^{--}, g^{--}\right\rangle$;

3. yet, $\left\langle M^{--}, g^{--}\right\rangle$is not isometric to $\left\langle M^{-+}, g^{-+}\right\rangle$, and

4. $\left\langle M^{++}, g^{++}\right\rangle$is not isometric to $\left\langle M^{+-}, g^{+-}\right\rangle$.

The above non-isometric extensions of Taub spacetime provide evidence for indeterminism in the sense of Butterfield's (1989) definition of determinism, which is a universe-based analysis, tailored to applications to GR. It says:

A theory with models $\left\langle M, O_{i}\right\rangle$ is $\mathbf{S}$-deterministic, where $\mathbf{S}$ is a kind of region that occurs in manifolds of the kind occurring in the models, iff: given any two models $\left\langle M, O_{i}\right\rangle$ and $\left\langle M^{\prime}, O_{i}^{\prime}\right\rangle$ containing regions $S, S^{\prime}$ of kind $\mathbf{S}$ respectively, and any diffeomorphism $\alpha$ from $S$ onto $S^{\prime}$ :

if $\alpha^{*}\left(O_{i}\right)=O_{i}^{\prime}$ on $\alpha(S)=S^{\prime}$, then:

there is an isomorphism $\beta$ from $M$ onto $M^{\prime}$ that sends $S$ to $S^{\prime}$, i.e. $\beta^{*}\left(O_{i}\right)=O_{i}^{\prime}$ throughout $M^{\prime}$ and $\beta(S)=S^{\prime}$.

Here $O_{i}$ stand for geometric object fields that either are definable in terms of a spacetime's metric, or characterise the matter fields of the spacetime. In our (vacuum) case the definition simplifies considerably, since in the absence of objects not definable in terms of a metric, the notion of isomorphy coincides with that of isometry, thus $\beta$ can be an isometry and the condition on $\alpha^{*}$ above concerns only objects defined in terms of a metric.

To check that the definition yields the verdict of indeterminism of GR, note that spacetime $\left\langle M^{++}, g^{++}\right\rangle$contains region $S^{++}=\Lambda^{++}[M]$, and spacetime $\left\langle M^{+-}, g^{+-}\right\rangle$contains region $S^{+-}=\Lambda^{+-}[M]$, where $\Lambda^{a b}: M \rightarrow M^{a b}$ is an embedding, which ensures that $\left\langle M^{a b}, g^{a b}\right\rangle$ is an extension of $\langle M, g\rangle$. For diffeomorphism $\alpha$ we take $\alpha=\Lambda^{+-}\left(\Lambda^{++}\right)^{-1}: S^{++} \rightarrow S^{+-}$. Then the push-forward $\alpha^{*}$ induced by $\alpha$ satisfies $\alpha^{*}\left(g^{++}\right)=g^{+-}$(by the condition on embedding), and hence $-\alpha^{*}\left(O_{i}\right)=O_{i}^{\prime}$ for any object field defined in terms of the metric. On the other hand, however, $\left\langle M^{++}, g^{++}\right\rangle$and $\left\langle M^{+-}, g^{+-}\right\rangle$are not isometric, by Chruściel and Isenberg's result quoted above. It follows that GR is indeterministic.

Let us next turn our attention to the evolution of individual objects (mass-less and massive particles) in GR. A notion relevant to a particle's evolution in GR is that of (null or timelike) geodesics, which are explained as trajectories of unaccelerated probe particles. Here "probe" means that the particles do not alter the geometry of the spacetime they move in. By EFE, a particle's motion is governed by the spacetime's metric, but the metric is influenced by the particle's motion as well. However, the influence of probe particles on the spacetime's metric is assumed to be negligible; the probe particle is thought of as moving in the background (independent of it) metric. But what are the trajectories of "real" particles in GR? A dominant tradition, going back to Einstein and Grossmann (1913), assumes that particles of sufficiently small mass and size move along geodesics. The tradition is supported by topological theorems to the effect that, given certain idealisations are assumed, the particle moves along a 
geodesic. A theorem to this effect is in Ehlers and Geroch (2004); the paper also gives reference to earlier results of this sort. ${ }^{18}$ In what follows, we thus consider a Taub spacetime inhabited by only such particles that satisfy the required idealisations. In this case all individual objects can be safely assumed to move along geodesics.

Suppose thus that a photon moves along a null geodesic in Taub spacetime. This spacetime has two non-isometric extensions, $\left\langle M^{++}, g^{++}\right\rangle$and $\left\langle M^{+-}, g^{+-}\right\rangle$. What happens to the photon as it leaves the initial region? That is, what does the photon's geodesic look like as the photon leaves Taub spacetime and proceeds to a new region of one of the two extensions? The answer is that there are two classes of null geodesics in Taub spacetime; geodesics of the first class are completed in one extension and geodesics of the other class-in the second extension [cf. Hawking and Ellis (1973, pp. 170-178) and Chruściel and Isenberg (1993, Lemma 3.2)]. Thus, the photon's evolution appears predestined: depending on which class the photon's geodesic belongs to, it will continue to one extension or the other.

One can get a better insight into the case at hand by considering the modal representation of the extensions of Taub spacetime. To obtain this modal representation, one glues together all $\left\langle M^{a b}, g^{a b}\right\rangle(a, b \in\{-,+\})$ into non-Hausdorff manifold $\tilde{M}=\left\langle M^{++}+M^{+-}+M^{-+}+M^{--}\right\rangle$; each $\left\langle M^{a b}\right\rangle$ comes out as a Hausdorff submanifold of it.

A photon traveling through the image of Taub spacetime in the modal representation $\tilde{M}$ moves along a null-geodesic. If it has two possible evolutions, say to (the images of) $\mathrm{M}^{++}$and $M^{+-}$, respectively, its geodesic must split, one path continuing in one extension and the other in the second extensions. That is, for the photon's indeterministic evolution, there should be two geodesics that coincide in a region of (the image of) Taub spacetime, but then continue separately, each in the (image of) of different $M^{a b}$. Note that the fact that two geodesics coincide in a region implies that the geodesics intersect at a point and their tangent vectors at that point agree.

Can such bifurcating geodesics occur in a Hausdorff manifold (or in a nonHausdorff manifold)? The following relevant fact obtains in a manifold (Hausdorff or not), equipped with a metric:

Fact 1 If a metric $g$ is appropriately continuous, given a point $p$ and a vector at this point, in some neighbourhood of p there is a unique geodesic that passes through the point and whose tangent at this point coincides with the vector. ${ }^{19}$

Note that the fact concerns only the local existence and uniqueness of a geodesic. However, for a Hausdorff manifold $M$, this local result can be strengthened to a global property: given the continuity condition on $g$, a point $p \in M$ and a vector $\xi$ at $p$, there is a unique maximal geodesic $\gamma: I \rightarrow M$ such that (1) $\gamma(0)=p$ and (2) $\dot{\gamma}(0)=\xi$, where $\dot{\gamma}(0)$ stands for a tangent to $\gamma$ at point $p=\gamma(0)$. Maximality of $\gamma$ means that if there is some other geodesic $\gamma^{\prime}: I^{\prime} \rightarrow M$ satisfying conditions

\footnotetext{
18 Of course, a direct way to learn a particles trajectory is to find an exact solution to a (relevant ) problem of motion of GR, yet there are only very few exact solutions of this kind.

19 See e.g. Chruściel (2011, p. 6). Typically, a metric of a GR spacetime meets the mentioned continuity requirement. For examples of metrics that do not satisfy it and accordingly generate non-unique bifurcating geodesics, see (Chruściel 1991, Appendix F).
} 
analogous to (1) and (2) above, then $I^{\prime} \subseteq I$. In sum, (given the continuity assumption on $g$ ), no geodesics can bifurcate in Hausdorff manifolds, but non-Hausdorff manifolds might be conductive to bifurcating geodesics. One might thus hope that in the nonHausdorff modal representation $\tilde{M}$ there is room for bifurcating geodesics, and hence, for alternative possible evolution. This hope is to be shattered.

To begin, since Taub spacetime (and hence its image by an isometry) is Hausdorff, a photon's geodesic in (the isometric image of) Taub spacetime is unique. Thus, if a bifurcation occurs, it must occur on a boundary between (the image of) Taub spacetime and the new regions of the extensions. What should this boundary look like to enable bifurcation? The answer is related to a failure of the Hausdorff property: for this to be possible, as the photon approaches the boundary, its geodesic should approach a non-Hausdorff pair of points, (i.e., a pair witnessing the non-Hausdorffness of the manifold), such that each point of the pair belongs to the new region of a different extension. More technically, in the modal representation, the intersection of the geodesic with (the image of) Taub spacetime should not have a supremum; rather there should be two (or more) minimal elements of the set of its upper bounds-these elements are witnesses for non-Hausdorffness.

Now, the surprising feature of the construction of Taub-NUT extensions (shared by constructions of Misner extensions or extensions of a polarised Gowdy spacetime) is that no geodesic approaching the boundary faces a non-Hausdorff pair of points. There is no bifurcation of geodesics, accordingly. One may wonder where are these non-Hausdorff pairs located in the modal representation for the extensions of Taub spacetime? To hint at a diagnosis, consider two geodesics traveling through (the image of) Taub spacetimes towards different extensions. (We know from the above that in these circumstances they can cross at a point but cannot coincide over an extended interval.) As such geodesics cross the boundary between Taub spacetime and the new region of a respective extension, they define two points. Now, no matter which two geodesics of this sort one takes, they define the pair of points that is a non-Hausdorff pair. Thus, in order to have a non-Hausdorff pair, we need to consider two geodesics separated, rather than coinciding in Taub spacetime. But such non-Hausdorff pairs are not conductive to bifurcation. ${ }^{20}$

To sum up, no photon's geodesics can bifurcate in the modal representation of the extensions of Taub spacetime. This result carries over to all massless and massive particles. Since geodesics in the modal representation represent possible evolutions of individual objects, no individual object in Taub spacetime has multiple alternative possible evolutions that consist in traveling to non-isometric extensions of that spacetime. Accordingly, Taub spacetime together with its non-isometric extensions is not a case of individuals-based indeterminism.

Arguably, the same verdict can be reached by Sattig's definition of "Strong Qualitative Determinism" (see Sect. 2.2), provided that an object's qualitative description is encoded by the object's geodesic. For possible worlds we take extensions $\left\langle M^{a b}, g^{a b}\right\rangle$ of Taub spacetime. We focus on a given $\left\langle M^{a b}, g^{a b}\right\rangle$ and pick a particle $x$ in the image

20 One may further wonder how non-Hausdorff pairs so peculiarly located emerge. The answer is that the manifolds considered are constructed as quotient structures of some auxiliary manifolds with respect to an equivalence relation resulting from a problem's symmetry, cf. Hawking and Ellis (1973, pp. 170-173). 
$\Lambda^{a b}(M) \subset M^{a b}$ of Taub spacetime. The qualitative behaviour of $x$ in $\Lambda^{a b}(M)$ is given by its geodesic $\gamma$. Suppose now that in some other extension $M^{a^{\prime} b^{\prime}}$ there is a particle, which behaves in $\Lambda^{a^{\prime} b^{\prime}}(M) \subset M^{a^{\prime} b^{\prime}}$ exactly like $x$ in $\Lambda^{a b}(M)$. This means that its geodesic $\gamma^{\prime}$ in the image of Taub spacetime must coincide with geodesic $\gamma$ of $x$ in the respective image of Taub spacetime. In mathematical parlance, $\left(\Lambda^{a b}\right)^{-1}[\gamma]$ and $\left(\Lambda^{a^{\prime} b^{\prime}}\right)^{-1}\left[\gamma^{\prime}\right]$ must denote one and the same geodesic in $M$. As we argued above, this geodesic in $M$ has a unique maximal extension, and this extension belongs to just one extended spacetime $\left\langle M^{a b}, g^{a b}\right\rangle$. Thus, there are no two particles, each belonging to a separate extended spacetime, whose qualitative descriptions match in (corresponding images of) Taub spacetime, but do not match elsewhere.Thus, as far as the extensions of Taub spacetime go, GR is Strongly Qualitatively Deterministic.

\section{Conclusions}

We described two traditions of thinking about determinism and indeterminism: the dominant tradition analyses these concepts in terms of global notions, like possible worlds or models of a theory, whereas the minority view defines them in terms of the possible behaviour of individual objects. We revisited a clash between these traditions that issued in the context of analysing systems whose initial segments are symmetric: counterintuitively such systems are diagnosed as deterministic by the dominant approach. We described how, by paying attention to possible evolutions of individual objects, the minority approach attempted to deliver an opposite verdict.

Next we provided an outline of an analysis of individuals-based determinism, the essence of which is the concept of modal representation of a system of a given theory. The modal representation for a given system contains multiple solutions (for fixed initial conditions) to the theory's basic equations, if there are such multiple solutions. An important structure, as it signals the presence of alternative possible evolution, is a modal fork. A theory is said to be indeterministic iff the modal representation of one of its systems contains a modal fork, otherwise a theory is said to be deterministic. Further, a theory is indeterministic individuals-wise iff a modal fork in a modal representation of that theory is due to the behaviour of an individual object admitted by the theory.

Finally, we exhibited a case from current physics, in which the universe-based approach and the individuals-based approach deliver opposite verdicts concerning determinism. The non-isometric extensions of Taub spacetime are viewed as indeterministic by the received (universe-based) approach, but, since no individual object has alternative possible evolutions in this system, the system is judged as deterministic by the individuals-based approach.

With this clash of verdicts in sight, imagine Laplace's demon residing in Taub spacetime. As it looks to the future, the demon perceives two dissimilar possible extensions of the spacetime. The demon realises that no matter how much it knows about Taub spacetime (in which it resides), it cannot learn which of these extensions will come to pass. The demon concedes that "the future is not present before its eyes", that is, Taub spacetime is indeterministic. Next the demon turns to small objects in Taub spacetime, all those little guys, photons, protons, cloaks, ships, sailors and the like. As the spacetime has multiple extensions, some individual objects should face 
multiple evolutions, like the cloak from Aristotle's story that might be cut up as well as wearing out first. The demon contemplates the physics of Taub spacetime and its extensions and learns that, given the position and the speed of each object, the object has exactly one possible evolution. No individual object faces multiple possible evolutions. Aristotle's cloak does not fit Laplace's demon.

Acknowledgements I am grateful to Thomas Müller for his detailed comments on an earlier version of this paper. I would also like to thank Oliver Pooley, Juliusz Doboszewski, Leszek Wroński, Jacek Wawer, Tadeusz Chmaj, Piotr Bizoń, and the audiences of the conference of the British Society for the Philosophy of Science in 2015 and of the meeting of the Budapest-Kraków research group in September 2014. Research leading to these results have received funding from the Foundation for Polish Science, research Grant "Mistrz 5/2011". The support of the joint Project of the Polish and Hungarian Academies of Science "Probability, Causality and Determinism" is as well gratefully acknowledged.

Open Access This article is distributed under the terms of the Creative Commons Attribution 4.0 International License (http://creativecommons.org/licenses/by/4.0/), which permits unrestricted use, distribution, and reproduction in any medium, provided you give appropriate credit to the original author(s) and the source, provide a link to the Creative Commons license, and indicate if changes were made.

\section{References}

Al-taee, M. (2010). Buckling of columns: Lecture notes of mechanics of solids, Chap. 9. http://web. aeromech.usyd.edu.au/AMME2301/Documents/Chapter09.

Belnap, N. (1992). Branching space-time. Synthese, 92, 385-434. 'Postprint' archived at PhilSci Archive, http://philsci-archive.pitt.edu/archive/00001003.

Belot, G. (1995). New work for counterpart theorists: determinism. The British Journal for the Philosophy of Science, 46, 185-195.

Belot, G. (2011). Background-independence. General Relativity and Gravitation, 43(10), 2865-2884.

Butterfield, J. (1989). The hole truth. The British Journal for the Philosophy of Science, 40(1), 1-28.

Butterfield, J. (2005). Determinism and indeterminism. In Routledge encyclopaedia of philosophy (Vol. 3). London: Routledge.

Choquet-Bruhat, Y., \& Geroch, R. (1969). Global aspects of the Cauchy problem in general relativity. Communications in Mathematical Physics, 14, 329-335.

Chruściel, P. (1991). On uniqueness in the large of solutions of Einstein's equations ("Strong Cosmic Censorship"). In Proceedings of the CMA, Canberra.

Chruściel, P. (2011). Elements of causality theory. arXiv:1110.6706v1 [gr-qc].

Chruściel, P., \& Isenberg, J. (1993). Nonisometric vacuum extensions of vacuum maximal globally hyperbolic spacetimes. Physical Review D, 48(4), 1616-1628.

Earman, J. (1995). Bangs, crunches, whimpers, and shrieks. Singularities and acausalities in relativistic spacetimes. New York: Oxford University Press.

Earman, J. (2008). Pruning some branches from Branching Spacetimes. In D. Dieks (Ed.), The ontology of spacetime II: Philosophy and foundations of physics (pp. 187-205). Amsterdam: Elsevier.

Ehlers, J., \& Geroch, R. (2004). Equation of motion of small bodies in relativity. Annals of Physics, 309(1), 232-236.

Einstein, A., \& Grossmann, M. (1913). Entwurf einer verallgemeinerten Relativitätstheorie und eine Theorie der Gravitation. Zeitschrift für Mathematik und Physik, 62, 225-261.

Hawking, S. W., \& Ellis, G. F. R. (1973). The large scale structure of space-time. Cambridge: Cambridge University Press.

Hodges, W. (1993). Model theory. Cambridge: Cambridge University Press.

Lewis, D. (1983). New work for a theory of universals. Australasian Journal of Philosophy, 61, 343-377.

Łukasiewicz, J. (1961). O determinizmie (On determinism). In Z zagadnień logiki i filozofii. Pisma wybrane (Selected Papers on Logic and Philosophy) (pp. 114-126). PWN, Warszawa. English translation, "On determinism", in Polish Logic, 1920-1939, S. McCall. (Ed.) (1967) Oxford: Clarendon.

Malament, D. (2012). Topics in the foundations of general relativity and Newtonian gravitation theory. Chicago: University of Chicago Press. 
Melia, J. (1999). Holes, haecceitism and two conceptions of determinism. The British Journal for the Philosophy of Science, 50(4), 639-664.

Müller, T. (2014). Alternatives to histories? Employing a local notion of modal consistency in branching theories. Erkenntnis, 79(3), 343-364.

Müller, T., \& Placek, T. (2015). Defining determinism. The British Journal for the Philosophy of Science. Advance Access, doi:10.1093/bjps/axv049.

Newman, E., Tamburino, L., \& Unti, T. (1963). Empty-space generalization of the Schwarzschild metric. Journal of Mathematical Physics, 4, 915-925.

Norton, J. D. (2008). The dome: An unexpectedly simple failure of determinism. Philosophy of Science, $75,786-799$.

Penrose, R. (1969). Gravitational collapse and spacetime singularities-The role of general relativity. La Rivista del Nuovo Cimento, 1, 252-276.

Placek, T. (2014). Branching for general relativists. In Müller, T. (Eds.), Nuel Belnap on indeterminism and free action (pp. 191-221). New York: Springer. Outstanding Contributions to Logic 2.

Placek, T., Belnap, N., \& Kishida, K. (2014). On topological aspects of indeterminism. Erkenntnis, 79, 403-436.

Prior, A. N. (1962). Limited indeterminism. The Review of Metaphysics, 16(1), 55-61.

Ringström, H. (2009). The Cauchy problem in general relativity. Zürich: European Mathematical Society Publishing House.

Rosales, R. R. (2012). Problem set number 2. 18.385j/2.036jmit. http://math.mit.edu/classes/18.385/ WWW385_2012/ProblemSets/ProblemSet_2012_02.

Sattig, T. (2015). Pluralism and determinism. Journal of Philosophy, 111, 135-150.

Zhihong, X. (1992). The existence of noncollision singularities in Newtonian systems. Annals of Mathematics, 135(3), 411-468. 